Erwin Genge, Erlenbach/Bern: Glaziale Vorkommen im Niedersimmen- und Diemtigtal.

Die Erforschung der eiszeitlichen Verhältnisse in den Voralpen hat innert eines Jahrhunderts gewaltige Fortschritte gemacht. Viele unserer Alpentäler haben in dieser Hinsicht eingehende Beschreibungen erfahren. Vom Simmen- und Diemtigtal lagen bis jetzt nur Darstellungen von Teilgebieten und Einzelbeobachtungen vor. Mein Bestreben geht dahin, diese Lücken schließen zu helfen. Die Arbeiten sind jedoch noch nicht abgeschlossen.

Das rund $62 \mathrm{~km}$ lange Simmental verläuft von der Wasserscheide südwestlich Lenk bis etwas über die Mitte hinaus in S-N-Richtung, biegt hierauf in großem Bogen gegen Osten und nimmt im untern Abschnitt genaue W-E Richtung ein. Die Umbiegung bedeutet auch eine Klimagrenze. Meine Untersuchungen beschränken sich vorläufig auf das untere Teilstück, sowie auf das ganze Diemtigtal. Das erratische Material für die Lokalgletscher lieferten die Niesenzone, die Klippen-, Breccien- und Simmendecke (aufgeführt in der Reihenfolge ihrer Überlagerungen). Für den Simmegletscher bilden die Hornfluhbreccie (Untere Breccie der Brecciendecke) von Garstatt abwärts und der Nummulitenkalk aus der Wildhorndecke für das ganze Tal die beiden typischen Leitgesteine. Im Diemtigtal stammen die Endmoränen in ihrer überwiegenden Zahl aus dem Bühlstadium, einige wenige nur aus den Rückzugsstadien von Gschnitz und Daun. Es konnten 3 verschiedene Eisrandlagen des Diemtigtalgletschers festgestellt werden. Die ältesten diluvialen Ablagerungen befinden sich bei Wampflen im Talgrund (Einlagerungen von interglazialen Schottern) und zwischen Horboden und Oey/Diemtigen (Stauschotter der Kirel, welche Würmmoränen tragen). Die Ursache der Stauung war der Kandergletscher, der von unten her einen wahrscheinlich bis Erlenbach reichenden Eisarm ins Simmental sandte (Gasterngranitfunde). Die Resultate der Untersuchungen im untern Simmental (Grenze Reidenbach-Garstatt) decken sich in Bezug auf die Lokalgletscher mit denen aus dem Diemtigtal; es fehlt nur das Daunstadium. Die Zeitbestimmung der Rückzugsstadien geschah mit Hilfe der Schneegrenzenbestimmungen und der einstigen Schneegrenzensenkung. Da das Bühlstadium drei Zonen mit vermehrten Endmoränenbildungen aufwies, erachtete ich es als gegeben, es in ein frühes, mittleres und spätes Bühl zu unterteilen, trotzdem die Klimabesserung, als ganzes betrachtet, allmählich erfolgt ist. Vergleiche mit F. Nussbaum (Die eiszeitliche Vergletscherung des Saanegebietes, 1906), W. Hofmann und E. Brückner (Beobachtungen über Moränen im Bereich der Kaiseregg, 1904) und M. Welten (Über die Spät- und postglaziale Vegetationsgeschichte des Simmentals, 1952), zeigten in den Resultaten gute Übereinstimmung.

Die Terrassen sind im Niedersimmental recht gut ausgebildet. Es seien erwähnt: das Simmenfluhniveau (P. BEck: Grundzüge der Talbildung im Berner Oberland, 1921) auf 1400-1500 m ü. M. am Talausgang, das Burgfluhniveau auf 900-1000 m und die im Talgrund vorhandene, bedeutend weniger hervortretende Erlenbachterrasse auf $650 \mathrm{~m}$. Burgfluh und Erlenbachterrassen sind auf der Sonnseite für geschlossene Siedlungen benützt worden. Ausgesprochene Talstufen fehlen im Haupttal.

\title{
DIE SCHWEIZ I956 - EIN LANDESKUNDLICHER RÜCKBLICK
}

\section{P. KÖCHLI}

Witterungsverlauf: Insgesamt war die Witterung $1956 \mathrm{zu}$ kalt und zu naß. Besonders die Sommermonate verzeichneten wesentliche Wärmedefizite (Zentralschweiz und Tessin etwa $1^{\circ} \mathrm{C}$, Alpennordseite, Mittelland und Jura $1 \frac{1}{2}-2^{\circ} \mathrm{C}$, Wallis $2 \frac{1}{2^{\circ}} \mathrm{C}$ ), dagegen einen Überschuß an Niederschlägen. Sie erreichten in den nördlichen Alpentälern und im Engadin etwa 120$140 \%$ des Durchschnittes, im Mittelland und am Juranordfuß meist 160-180\%, im Gebiet des Kantons Schaffhausen und im Wallis etwas mehr als $200 \%$ und im nördlichen und mitt- 
leren Tessin ungefähr 250\%. Das Südtessin wies normale Niederschlagsverhältnisse auf. Eine Besonderheit war der äußerst kalte und trockene Februar auf der Alpennordseite, der kälteste seit Temperaturbeobachtungen aufgezeichnet werden. Die Abweichungen nach unten betrugen in den Niederungen bis $10^{\circ} \mathrm{C}$. Die Monats-Niederschläge machten in der Westschweiz nur ungefähr einen Zehntel des Mittels aus, in den Alpen etwa $25 \%$, in den östlichen Landesteilen rund $50 \%$.

Das Jahr 1956 wies außerordentlich starke Hagelschäden (62 Hageltage) auf, ein Drittel entfiel auf die Monate September und Oktober. Am 7. Juni wurden vor allem Gürbetal, Emmental, Entlebuch, Suhrental, Wynental, aargauisches Seetal und östliches Hallwilerseegebiet betroffen, im August das Mendrisiotto, das Weinbaugebiet zwischen Cully und Vevey, Gürbetal, Entlebuch, Bezirk St. Gallen und einzelne Landstriche im Kanton Thurgau. Am 1o. September entluden sich schwere Gewitter zwischen Lausanne und dem mittleren Broyetal, über dem Basler, Solothurner und Aargauer Jura, dem Seeland sowie dem Dreieck Napf-Sempacher-Zugersee. Rund 380 Gemeinden meldeten Schaden. Der letzte schwere Hagelschlag erfolgte am 5. Oktober im Tessin.

Wirtschaftsverlauf: Industriell herrschte wiederum Hochkonjunktur, während die Landwirtschaft unter den Folgen des Februars und des Sommers litt.

Landwirtschaftlich war 1956 mit wenigen Ausnahmen ein schlechtes Jahr. Der Gesamtindex für die Produzentenpreise landwirtschaftlicher Erzeugnisse betrug 1955: 98,9; 1956: 99,9 der Gesamtindex der Preise landwirtschaftlicher Produktionsmittel 1955: 107,o, 1956: 11o,4. Aufwand und Ertrag klaffen immer mehr auseinander, was im Bauernstand Beunruhigung und weitere Abwanderung zu andern Berufen bewirkt. Deshalb sind erneut Untersuchungen durchgeführt worden, wie vor allem den rund 82000 Bergbauernkleinbetrieben zu helfen sei. Ihre wichtigste Einnahmequelle ist Nutz- und Zuchtvieh. 1951 war der Anteil der Aufzuchttiere am Gesamtrindviehbestand in den Berggebieten 53,5\%, im Flach- und Hügelland $34,8 \%$. Wenn nun der Mittellandbauer sein Vieh selber aufzieht, was in den letzten Jahren vermehrt geschah, zerstört er die Existenzgrundlage des Bergbauern. Zum Vorteil von Berg- und Mittelbauer muß sich deshalb freiwillige Arbeitsteilung durchsetzen. Daneben sind vor allem Güterzusammenlegung und Heimarbeit zu fördern. Die Industrialisierung wirkt nicht in allen Beziehungen vorteilhaft (Sog der Industriearbeit, extensivere Bewirtschaftung des Bodens, Aufgabe des Bauernbetriebes).

Von der Anbaufläche des Wintergetreides (126000 ha) winterten 80000 ha aus und mußten neu angesät werden. Die Getreideanbaufläche wurde dadurch 1956 um 5000-10000 ha kleiner als 1955 (174750 ha). Dagegen nahm die Anbaufläche aller übrigen Kulturpflanzen ohne Raps um rund 4000 ha zu. Die Kartoffelernte betrug 19561570000 t oder 266 q/ha gegenüber 193 q/ha 1955. Die Zuckerrübenernte war die zweitgrößte seit dem Bestehen der Zuckerfabrik Aarberg und erreichte 234833 t bei einem durchschnittlichen Zuckergehalt von $15,90 \%$. Daraus konnten gut $30000 \mathrm{t} Z$ ucker gewonnen werden. Die Leistungsfähigkeit der Zuckerfabrik ist damit voll ausgeschöpft, so daß eine zweite in der Ostschweiz geplant wird. Die Rebfläche ist um 295 ha auf 12845 ha gesunken. Die Anbaufläche betrug in der deutschen Schweiz 1721 ha, in der Westschweiz 9344 ha, im Tessin und Misox 1780 ha. Der Ertrag belief sich auf 17877 q Tafeltrauben und 445 16o hl Wein (1955: 800966 hl). Die Hektarerträge erreichten durchschnittlich $34,6 \mathrm{hl}(1955: 63,8 \mathrm{hl})$, was die Februarkälte spiegelt. Eine Zählung der Nutztiere ergab (provisorisch):

\begin{tabular}{|c|c|c|c|c|c|c|c|c|c|c|c|c|c|}
\hline & & & & & & & & & & & & Verà & rung \\
\hline & & & & & & & & & & 1951 & 1956 & absolut & prozentual \\
\hline Rindvieh & & . . & . & . & . & . & . & . & . & 1607271 & 1645000 & 37729 & $+2,3$ \\
\hline Schweine & & . & . & . & . & . & . & . & . & 892092 & 1158700 & +266608 & $+29,8$ \\
\hline Ziegen & . & . . & . & . & . & . & . & . & . & 148242 & 112500 & $-\quad 35742$ & $-24,2$ \\
\hline Schafe & . & . . & . & . & . & . & . & . & . & 191736 & 200700 & 8964 & $+\quad 4,6$ \\
\hline Pferde & . & . . & . & . & . & . & . & . & . & 181374 & 166700 & $-\quad 14674$ & $-8,0$ \\
\hline Maultiere & & . . & . & . & . & . & . & . & . & 2156 & 1550 & 606 & $-28,1$ \\
\hline Esel . & . & . . & . & . & . & . & . & . & . & 393 & 370 & - & $-\quad 5,8$ \\
\hline Hühner & & . & . & . & . & . & . & . & . & 6239881 & $6+02000$ & +162119 & $+2,5$ \\
\hline Gänse un & & Enter & & . & . & . & . & . & . & 68371 & 55100 & $-\quad 13271$ & $-19,4$ \\
\hline Bienenvöl & ker & r. & . & . & . & . & . & . & . & 339243 & 298300 & - 40943 & $-12,0$ \\
\hline
\end{tabular}

Als besonderes Problem stellt sich in der Schweiz stets das Verhältnis Ackerland zu Wiesland und Hackfruchtanbau (vor allem Zuckerrüben) zu Getreideanbau. In diesem Zusammenhang ist die Aufstellung über den prozentualen Anteil der Inlandproduktion am Bedarf aufschlußreich :

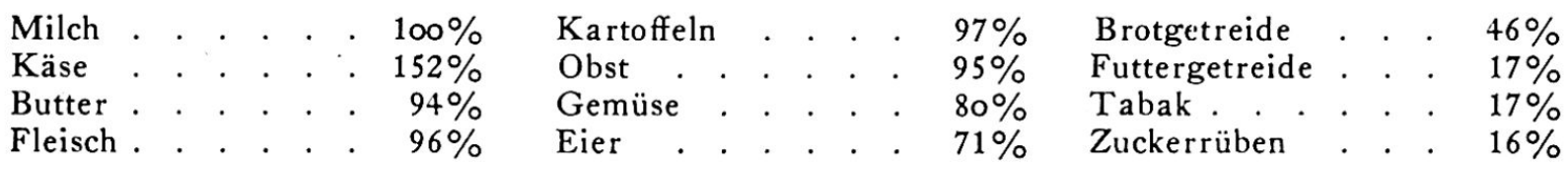


Eine etwas weiter reichende Änderung dieser Verhältnisse erwartet man vom Bau einer zweiten Zuckerfabrik und einer Ausdehnung der Zuckerrübenanbaufläche von 5900 auf rund 10000 ha. Dadurch könnten $25 \%$ des Zuckerverbrauches durch die Inlandproduktion gedeckt werden.

Land- und forstwirtschaftlich sind noch erwähnenswert:

Die Fertigstellung des drittgrößten Meliorationsunternehmens in der Schweiz im Klettgau (Linthebene und St. Galler Rheintal waren größer), wo 3200 ha Acker- und Wiesland und über 300 ha Rebland melioriert wurden;

die Gefahr einer Vernichtung der tessinischen Kastanienwälder durch den Rindenkrebs, gegen den man bis jetzt noch kein Abwehrmittel besitzt. Bereits sind größere Gebiete davon befallen;

die Wiederaufforstung des Wallbachgrabens und seines Einzugsgebietes von too ha im Simmental.

Die Industrialisicrung setzte sich fort; die Zahl der Fabrikbetriebe erreichte rund 12 oo (615 000 Arbeiter). Die größten Zunahmen erfolgten in Maschinenindustrie, Metallbearbeitung, Holzindustrie und Uhrenbranche.

Der Außcnhandel zeigt 1956 folgende Entwicklung:

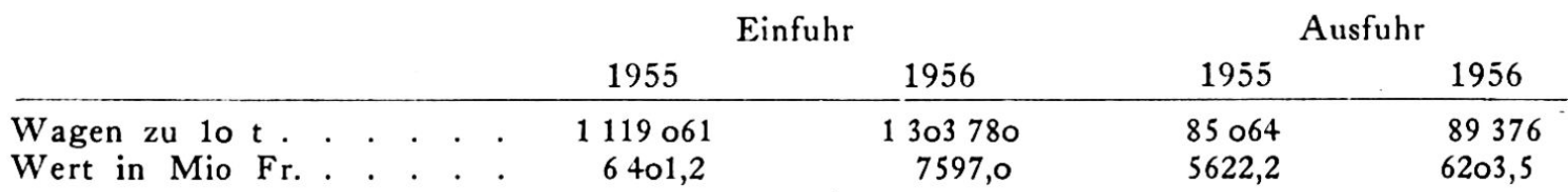

Defizit des Warenverkehrs: 1393,5 Mio Fr.

Der $A u ß c n h a n d c l$ weist folgende räumliche Verteilung und Verschiebungen auf:

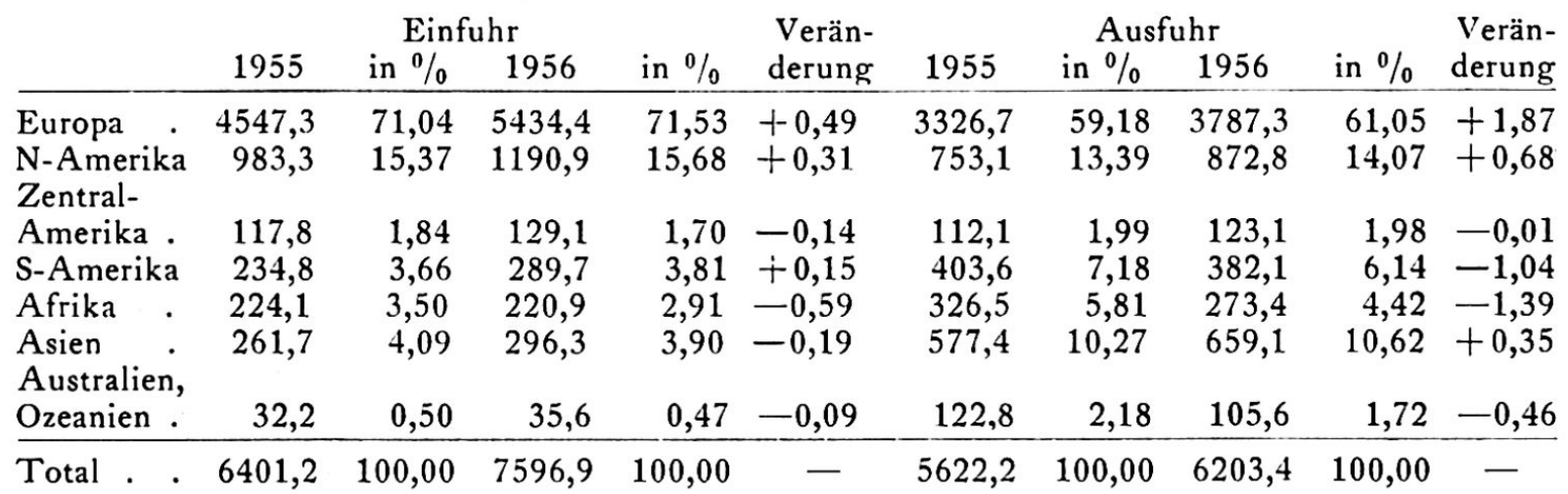

Der Handel mit dem europäischen Wirtschaftsraum hat sich erneut verstärkt. Beachtliche Mehrimporte erfolgten aus Westdeutschland, USA, Italien, Benelux, Großbritannien und Frankreich. Vermehrt konnte nach Frankreich, USA, Westdeutschland, den Niederlanden, Italien, Benelux, China und Indien verkauft werden.

Elektrizitätswirtschaft (Hydrographisches Jahr: 1. Oktober 1955 bis 3o. September 1956): Erzeugung und Verbrauch zeigen folgende Entwicklung:

a) Erzeugung:

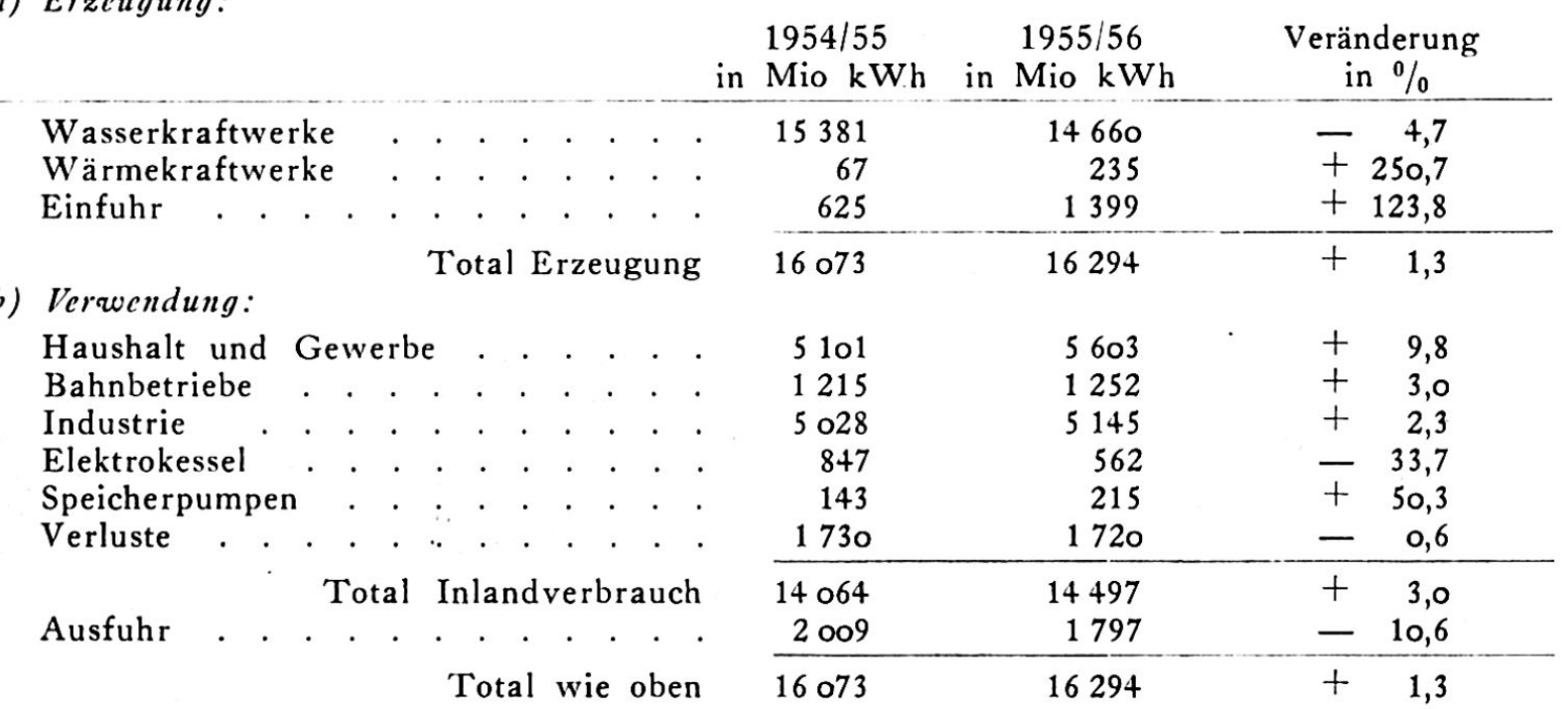


Die starke Zunahme bei den Wärmekraftwerken und bei der Einfuhr ist auf den Februar zurückzuführen.

1956 wurden folgende Kraftwerkbauten begonnen: Ackersand II der Lonzawerke, Blenio, Diablerets-Lac d'Armon, Hinterrhein, Kirel-Filderich (Simmental), Lostallo (Monte FornoBodio) und Sedrun-Tavanasa.

Verkehr: Die Bundesbahnen verzeichneten einen Transportrekord, von 215,7 Mio Personen (1955: 208,4 Mio) und 24,75 Mio t Gütern (1955: 23,47 Mio t).

Der Straßenverkehr stieg erneut sprunghaft. Der Motorfahrzeugbestand am 3o. September 1956 war:

\begin{tabular}{|c|c|c|c|c|c|c|c|c|c|}
\hline & & & & & & & 1955 & 1956 & Veränderung in $\%$ \\
\hline Autobusse unc & & Autoc & cars & . & . . & . & $25 n 2$ & 2594 & $+3,6$ \\
\hline Personenwager & & . . & . . & . & . . & & 27 o 821 & 308679 & $+13,9$ \\
\hline Personenwager & $n f$ & für & W.ar & ent & ransp & orte & 8696 & 11392 & $+31,0$ \\
\hline Lieferwagen & 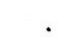 & . . & $\cdot$. & . & . . & . . & 15883 & 16534 & $+\quad 4,1$ \\
\hline Lastwagen & 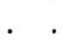 & ${ }^{\circ}$ & . . & . & . . & . . & $26 \circ 38$ & 28075 & $+7,8$ \\
\hline Spezialwagen & . & . . & . & . & . . & . . & 2801 & 3188 & $+13,8$ \\
\hline Gewerbliche T & Tral & ktorer & & . & . . & . . & 1149 & 1174 & $+\quad 2,1$ \\
\hline Motorfahrräde & & . . & . & . & . . & . . & 59201 & $747 \circ 2$ & $+26,2$ \\
\hline Roller.. & . & . & . & . & . . & . & 76654 & 81960 & $+\quad 6,9$ \\
\hline Motorräder &.$\quad$. & . . & . & . & .. & . . & 80586 & 78454 & $-2,7$ \\
\hline & $\mathrm{M}$ & Sotorf & fahr & zeug & ge $\mathrm{T}$ & otal & 544331 & 606752 & $+11,4$ \\
\hline
\end{tabular}

Der steigende Straßenverkehr (gut 2 Mio einreisende ausländische Fahrzeuge) ließ die Aussprache um die Planung des Straßennetzes inkl. Alpendurchstiche nicht ruhen. Ende 1956 hatte die Planungskommission ihre Vorschläge weitgehend bereinigt.

Auch der Flugverkehr ist sprunghaft angewachsen:

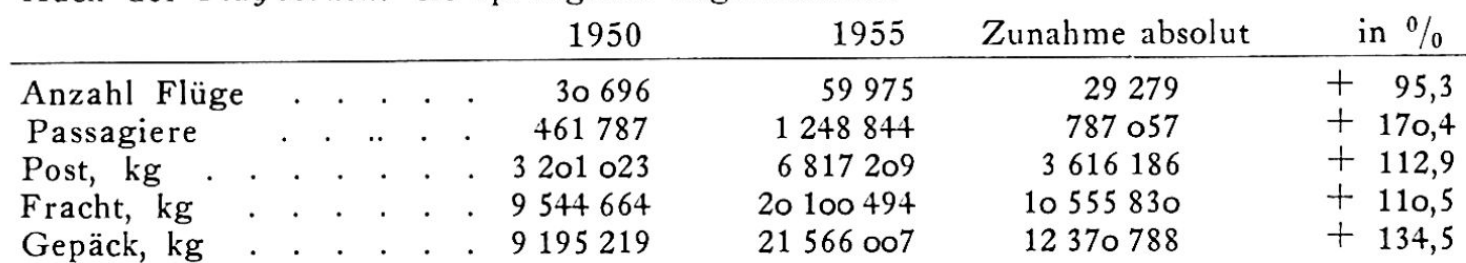

Die Entwicklung und die Umstellung im Flugverkehr nötigen die Flugplätze Zürich-Kloten und Genf-Cointrin, später auch Basel-Mülhausen wesentlich auszubauen, wobei zunächst Pistenvergrößerungen notwendig sind. Auch Bern prüft den Bau eines intereuropäischen Flugplatzes bei Herrenschwand, da das Belpmoos nicht mehr genügt.

Auch im Fremdenverkehr setzte sich die Aufwärtsentwicklung fort. Sowohl die Zahl der Gastbetten (1956: 19o 555, 1955: 186 o23) als die der Logiernächte (1956: 24243 674, 1955: 23635434 ) verzeichneten Zunahmen, während die Bettenbesetzung mehr oder weniger konstant blieb $(34,8 \%)$. Die Ausgaben der Ausländer stiegen von rund 99o Mio auf über $1 \mathrm{Mrd}$ Franken; der Aktivsaldo freilich dürfte infolge der Preissteigerungen kaum eine Veränderung erfahren haben.

Hinsichtlich der Bautätigkeit wurden bei 464 erfaßten Gemeinden mit mehr als $2000 \mathrm{Be}-$ wohnern (Städten) 31188 Neubauwohnungen ermittelt (1955: 31 320). Sie entfielen hauptsächlich auf die kleinen und die Großstädte. Ein leichter Rückgang ergab sich bei den Ein- und eigentlichen Mehrfamilienwohnungen. Das Wohnungsangebot belief sich auf 23200 gegen 24500 im Vorjahre. Die Gebäude ohne Wohnungen erfuhren einen Zuwachs von 6104 auf 6627 Einheiten, insbesondere Fabriken, Garagen, Schulen usw. Insgesamt hätte damit also eine Bauvolumenvermehrung stattgefunden, die etwa einer Stadt von 100-120 ooo Einwohnern (Lausanne: 1950: 33945 Wohnungen, 106807 Einwohner) entspräche. Die letzten 1o Jahre sahen einen mittleren Wohnungszuwachs von je 20000 Einheiten, was einen jährlichen Neubau von 5-7 Zehntausenderstädten ausmachte.

Verschicdenes: Die Planung für die Juragewässerkorrektion wurde vorwärts getrieben. Bei Eglisau erbohrte man in verschiedenen Tiefen ertragreiche Mineralquellen. In $360 \mathrm{~m}$ Tiefe traf man auf eine subthermale Schwefelquelle. Das Oberengadin wird seit Jahren wieder von Überschwemmungen heimgesucht. Am meisten betroffen sind Celerina/Schlarigna, Samedan, Bever und La Punt. Am bedrohlichsten sind die Hochwasser aus dem schuttreichen Roseggtal. Größere Rutschungen ereigneten sich im Zusammenhang mit den reichlichen Niederschlägen im Traverstal.

Ausblick fïr 1957: Die Industrialisierung, der Wohnungsbau und der Ausbau des Straßennetzes gehen weiter. Sie beanspruchen jährlich etwa 2000 ha Kulturland und Wald. Land- 
schaftliche Veränderungen werden sich wiederum vor allem durch den Kraftwerkbau einstellen, ferner durch Aufforstungen in Gebirgsgegenden (Bannwälder) und durch den andauernden Rückzug der Gletscher.

Qucllen: Die Volkswirtschaft 1956/57, Berichte der Schweiz. Bankgesellschaft, der Schweiz. Kreditanstalt, Bulletin des SEV 1956, Energie-Konsument 1956, führende Tageszeitungen u. a.m. Ferner sei zahlreichen eidgenössischen und kantonalen Ämtern für bereitwillige Auskunft bestens gedankt.

\author{
DIE SOWJETUNION I 956 \\ Ein landeskundlicher Rückblick im Lichte der ncusten Statistik
}

\title{
E. WINKLER
}

Bis vor kurzem waren die Möglichkeiten, sich über die Sowjetunion der Gegenwart anhand authentischer Daten zu orientieren sehr gering; der Westen war auf Schätzungen aus spärlichen Zeitungsmeldungen oder auf nicht weniger kursorische Angaben in Parteireden angewiesen. Im Jahre 1956 gab nun die Statistische Zentralverwaltung beim Ministerrat der UdSSR ein Sammelwerk in Form der westlichen statistischen Jahrbücher heraus, das erlaubt, sich ein etwas zuverlässigeres Bild vieler Bereiche der Bevölkerung, Wirtschaft und Kultur zu machen. Es soll im folgenden versucht werden, aus dem Buche, das kürzlich dankenswerterweise auch in deutscher Übersetzung erschien* einige der bemerkenswertesten Zahlen zusammenzustellen, vor allem im Blick darauf, daß der Westen an einer möglichst objektiven Kenntnis der Sowjetunion interessiert sein muß.

Arcal und Bcvölkcrung. Der Bund der Sozialistischen Sowjetrepubliken hat seine Fläche in den letzten fünfzig Jahren mehrfach, wenn auch keineswegs grundlegend verändert: hatte das Zaristische Rußland 1913 ein Areal von 22,3 Mio km², so die UdSSR 1918/39 infolge der Loslösung namentlich der Baltischen Provinzen und Finnlands nur noch 21,7 Mio. Der zweite Weltkrieg trug ihr erneut größere Ländereien ein, so daß sie seit 194522,4 Mio $\mathrm{km}^{2} \mathrm{gro}$ ist. Auf dieser Fläche erfuhr das Land eine Bevölkerungszunahme von 165,7 (1913) auf 200,2 Millionen Personen (April 1956), was einem Zuwachs von knapp $26 \%$ entspricht (18971956: 94 Mio oder 89\%). Im Jahre erfolgte demnach im Mittel ein Zuwachs von 1,5\%, was in Anbetracht der bevölkerungspolitischen Perioden der beiden Weltkriege (Verluste im 2. Weltkrieg nach sowjetischen Angaben 16 Mio) und der Revolution dazwischen wohl als außerordentlich zu bezeichnen ist und jedenfalls die durchschnittliche Bevölkerungszunahme der europäischen Staaten mit Ausnahme der Niederlande $(2,1 \%$, Griechenland $4,8 \%)$ erheblich übertrifft. Die sowjetische Schätzung für 1956 steht freilich in einem gewissen Widerspruch mit frühern Angaben von Führern des Landes, nach denen die Gesamtzahl heute eigentlich 210220 Mio betragen sollte (die übrigens das Ausland in der Regel angenommen hatte). Auch die von der offiziellen Statistik angegebenen Zuwachsquoten machen eher die größere Ziffer wahrscheinlich; doch muß wohl eine neue Volkszählung abgewartet werden, um in dieser Hinsicht klar zu sehen.

Auf jeden Fall scheinen die Völker der UdSSR, die um 1900 noch eine Geburtenquote von gut 50 pro 1000 hatten, aus zahlreichen Gründen, an Geburtenfreudigkeit entschieden eingebüßt zu haben. Nur aus dem — freilich in vielen Ländern analogen — auffälligen Rückgang der Sterbequote läßt sich verstehen, daß der Geburtenübcrschuß in den letzten fünfzig Jahren auf beinahe gleicher Höhe blieb, was folgende Tabelle veranschaulicht: Auf 1000 Einwohner wurden registriert:

\begin{tabular}{cccc} 
im Jahr & Geburten & Sterbefälle & Geburtenüberschuß \\
\hline 1897 & 50,4 & 33,1 & 17,3 \\
1913 & 47,0 & 30,2 & 16,8 \\
1926 & 44,0 & 20,3 & 23,7 \\
1940 & 31,7 & 18,3 & 13,4 \\
1950 & 26,5 & 9,6 & 16,9 \\
1953 & 24,9 & 9,0 & 15,9 \\
1955 & 25,6 & 8,4 & 17,2
\end{tabular}

* Die UdSSR in Zahlen. Moskau bzw. Berlin (Verlag Die Wirtschaft) 1956. Dazu : J. SiEMER : Bevölkerungs- und raumpolitische Entwicklungen in der Sowjetunion. Informationen des Instituts für Raumforschung, Godesberg 1956, 433-442. Sonderheft Stadtgeographie der Geogr. Nachrichten Nr. 38, 1956, Moskau, russisch). Obolenski, K.: Die Stärke der Kolchose... Fragen der Wirtschaft (Nr. 10, 1956, Moskau, russisch). Baranski, N. N.: Ökonomische Geographie der Sowjetunion. Berlin 1954 (14. Aufl.). Sauschkin, J. G.: Zur Entwicklung und geographischen Verteilung der Industrie der UdSSR im 6. Planjahrfïnft (1956-1960). Geographie in der Schule (1956, H. 2). 